\title{
Spectroscopic measurement of an atomic wave function
}

\author{
Kishore T. Kapale, ${ }^{1}$ Shahid Qamar, ${ }^{1,2}$ and M. Suhail Zubairy ${ }^{1,3}$ \\ ${ }^{1}$ Institute for Quantum Studies, and Department of Physics, Texas A\&M University, College Station, Texas 77843-4242 \\ ${ }^{2}$ Department of Physics and Applied Mathematics, Pakistan Institute of Engineering and Applied Science, Nilore, Islamabad, Pakistan \\ ${ }^{3}$ Department of Electronics, Quaid-i-Azam University, Islamabad, Pakistan
}

(Received 19 August 2002; published 10 February 2003)

\begin{abstract}
We present a simple spectroscopic method based on Autler-Townes spectroscopy to determine the centerof-mass atomic wave function. The detection of spontaneously emitted photons from a three-level atom, in which two upper levels are driven by a classical standing light, yields information about the position and momentum distribution of the atom [A. M. Herkommer, W. P. Schleich, and M. S. Zubairy, J. Mod. Opt. 44, 2507 (1997)]. In this paper, we show that both the amplitude and phase information of the center-of-mass atomic wave function can be obtained from these distributions after a series of conditional measurements on the atom and the emitted photon.
\end{abstract}

DOI: 10.1103/PhysRevA.67.023805

PACS number(s): 42.50.Ct, 42.50.Vk, 03.75.Be, 03.75.Dg

\section{INTRODUCTION}

Preparation and measurement of a quantum state have been an avenue of great interest ever since the advent of quantum mechanics [1]. There has been an upsurge of activity in the field in recent years [2]. Preparing a quantum state is probably the easier task of the two; once the observable property required is chosen, a measurement of this observable delivers the required quantum state, through so-called "collapse" of the wave function. However, to determine the complete quantum state of a system one has to perform a whole set of measurements, as a single measurement reveals only a particular aspect of the state. Thus, in general, quantum state measurement is an immensely complicated endeavor.

Various interesting proposals have been made to measure the quantum states of light as well as matter. Majority of the methods for both kinds of wave functions are based on the measurement of Wigner function that bears a close connection with the density operator of the system [3], thus characterizing the quantum state completely. There are also several other techniques outside this tomographic $[4,5]$ arena for measurement of the quantum state of the radiation field: These include methods based on absorption and emission spectroscopy [6], conditional measurement of atoms in a micromaser cavity [7], and more [8]. Similar study of the matter wave field includes probing the quantum motion of the trapped atoms [9] or ions [10], or measurement of center-ofmass motion of an atomic beam in transverse as well as longitudinal direction, with the techniques used to probe the atoms being either tomographic or interferometric [11]. Experimentally, tomographic method has been applied to the vibrational state of a diatomic molecule [12] and interferometric method for the holographic reconstruction of molecular wave packets [13] and electronic Rydberg wave packets [14]. In essence, measurement of a quantum state of light or matter is possible by utilizing the interaction between the two and measuring the effect of it on one to predict the quantum state of the other.

Measuring the center-of-mass quantum state of an atomic beam is particularly of interest due to its possible applica- tions in the field of atom interferometry [15] and atom optics [16]. In this paper, we propose a possible experimental situation to measure both the amplitude and phase properties of the quantum character of the center-of-mass motion of an atomic beam. Our method is very different from either the interferometric or the tomographic method. It is primarily based on three interesting and well-known phenomena. The first one is Autler-Townes spectroscopy [17], which implies that the spontaneous emission spectrum of a driven threelevel atom shows splitting of the emission line. The second one is Autler-Townes microscopy [18], which suggests that this splitting depends on the position of the atom in the standing light field. The third one is that the detection of a spontaneously emitted photon from an atom passing through a standing light field localizes the atom [19]. Each atom from the beam is coupled to the cavity field through its internal degrees of freedom. Thus, the modified spontaneous emission spectrum has complete information about the center-ofmass wave function of the atomic beam. We determine the position and momentum distribution of this atomic beam conditioned on the detection of a spontaneously emitted photon. Thus, the atom is left in its internal ground state after the detection of the photon. We show that the amplitude and phase information of the center-of-mass wave function are contained in the measured position and momentum distributions, respectively.

\section{THE SCHEME}

The proposed experimental situation is illustrated in Fig. 1. A three-level atom interacts with a classical standing light field of wave vector $\kappa=2 \pi / \lambda=\omega_{a c} / c$ aligned along the $x$ direction. Here, we assume the transition $|c\rangle-|a\rangle$ of the three-level atom to be in resonance with the driving field and the transition $|a\rangle-|b\rangle$ to be coupled to the reservoir of vacuum modes $\{\mathbf{k}\}$, thus giving rise to spontaneous emission on that transition.

We assume that the atom is moving with a sufficiently high velocity such that its interaction with the driving field does not affect its motion along the $z$ direction, which can therefore, be treated classically. We also assume that the in- 


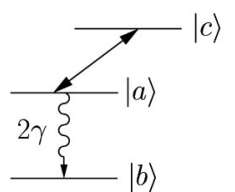

Energy levels



FIG. 1. Three-level atom interacting with a classical standing light field of wave vector $\kappa=2 \pi / \lambda=\omega_{a c} / c$ aligned along the $x$ direction. The transition $|c\rangle-|a\rangle$ of the three-level atom is in resonance with the driving field and the transition $|a\rangle-|b\rangle$ is coupled to the reservoir of vacuum modes $\{\mathbf{k}\}$, thus giving rise to spontaneously emitted photons that can be detected thereafter.

teraction time of the atom with the standing light field is sufficiently small. As a result, the transverse center-of-mass position of the atom does not change significantly compared to the wavelength of the field during the interaction time. Thus, we can neglect the kinetic energy $\hat{p}_{x}^{2} /(2 M)$ of the atom in the interaction Hamiltonian under the Raman-Nath approximation.

The interaction Hamiltonian, in the dipole, rotating wave, and the Raman-Nath approximations is given by

$$
\begin{aligned}
\hat{H}= & \hbar g(x)[|c\rangle\langle a|+| a\rangle\langle c|]+\hbar \sum_{\mathbf{k}}\left[g_{\mathbf{k}}(x) \mathrm{e}^{i\left(\omega_{a b}-\nu_{k}\right) t}|a\rangle\right. \\
& \left.\times\left\langle b\left|\hat{b}_{\mathbf{k}}+g_{\mathbf{k}}^{*}(x) \mathrm{e}^{-i\left(\omega_{a b}-\nu_{k}\right) t}\right| b\right\rangle\langle a| \hat{b}_{\mathbf{k}}^{\dagger}\right] .
\end{aligned}
$$

Here $g(x)=G \sin (\kappa x)$ is the position-dependent Rabi frequency, associated with the $|a\rangle-|c\rangle$ transition and the operators $\hat{b}_{\mathbf{k}}$ and $\hat{b}_{\mathbf{k}}^{\dagger}$ describe the annihilation and creation of photons in the reservoir modes with wave vector $\mathbf{k}$ and frequency $\nu_{\mathbf{k}}=c k$. The parameter $g_{\mathbf{k}}(x)=G_{\mathbf{k}} \exp (i k x \cos \theta)$ is the coupling strength associated with the spontaneous emission of a photon at an angle $\theta$ with respect to the $x$ axis and $\omega_{a b}$ is the transition frequency between the levels $|a\rangle$ and $|b\rangle$.

We would like to show that the spontaneously emitted radiation yields information about the atomic wave function. For this purpose, we solve the Schrödinger equation corresponding to the Hamiltonian given by Eq. (1). To start with we express the atom-field wave vector $|\psi(t)\rangle$ of the complete system as

$$
\begin{aligned}
|\psi(t)\rangle= & \int \mathrm{d} x f(x)|x\rangle\left[C_{a, 0}(x, t)|a\rangle|0\rangle+C_{c, 0}(x, t)|c\rangle|0\rangle\right. \\
& +\sum_{\mathbf{k}} C_{b, 1_{\mathbf{k}}}(x, t)|b\rangle\left|1_{\mathbf{k}}\right\rangle .
\end{aligned}
$$

Here, $C_{a, 0}(x, t)$ and $C_{c, 0}(x, t)$ represent the positiondependent probability amplitudes for the atom to be in the levels $|a\rangle$ and $|c\rangle$, respectively, with no photons in the reservoir modes and $C_{b, 1_{\mathbf{k}}}(x, t)$ is the probability amplitude for the atom to be in level $|b\rangle$ with one photon in mode $\mathbf{k}$. Note that $f(x)$ is the center-of-mass wave function of the atom.
Constructing the usual Schrödinger equation of motion for $|\psi(t)\rangle$ using the Hamiltonian (1) and splitting different internal state components, we arrive at the equations of motion for the probability amplitudes:

$$
\begin{gathered}
\dot{C}_{a, 0}=-\gamma C_{a, 0}-i g C_{c, 0}, \\
\dot{C}_{c, 0}=-i g C_{a, 0}, \\
\dot{C}_{b, 1_{\mathbf{k}}}=-i g_{\mathbf{k}}^{*} \mathrm{e}^{-i \delta_{k} t} C_{a, 0},
\end{gathered}
$$

where $\delta_{k}=\omega_{a b}-\nu_{k}$ and $2 \gamma$ is the spontaneous emission rate from level $|a\rangle$ to level $|b\rangle$.

Two of the above Eqs. (3) and (4) can be coupled together and solved easily to determine $C_{a, 0}$. With the assumption that the atom is initially in level $|a\rangle$, the solution for the amplitude $C_{a, 0}$ takes the form

$$
\begin{aligned}
& C_{a, 0}(x, t)=\mathrm{e}^{-\gamma t / 2}\left[\cosh \left(\frac{1}{2} \sqrt{\gamma^{2}-4 g^{2}(x)} t\right)\right. \\
& \left.-\frac{\gamma}{\sqrt{\gamma^{2}-4 g^{2}(x)}} \sinh \left(\frac{1}{2} \sqrt{\gamma^{2}-4 g^{2}(x)} t\right)\right] \text {. }
\end{aligned}
$$

This expression can in turn be substituted in Eq. (5), which after integration yields the desired expression for $C_{b, 1_{\mathbf{k}}}(x, t)$. Our aim is to obtain complete information about the wave function of the atom from the spontaneously emitted photon, therefore, we need to consider times which are large compared to the atomic decay time, i.e., $t \gg \gamma^{-1}$. This ensures that the photon has indeed been emitted. In this longtime limit, we arrive at the following steady-state value:

$$
C_{b, 1_{\mathbf{k}}}(x, \infty)=\frac{G_{\mathbf{k}}^{*} \mathrm{e}^{-i k x \cos \theta} \delta_{k}}{G^{2} \sin ^{2}(\kappa x)-\delta_{k}^{2}+i \delta_{k} \gamma} .
$$

All the information required to regenerate the center-ofmass atomic wave function is contained in the expression for the probability amplitude given by Eq. (7). We elaborate the details of how to extract this information in the following section.

\section{EXTRACTING THE AMPLITUDE AND PHASE INFORMATION OF THE CENTER-OF-MASS WAVE FUNCTION}

We now discuss how the conditional states of the system provide information about the center-of-mass wave function of the atom $f(x)$. The wave function $f(x)$ is in general complex and has both the amplitude and phase. One needs to deal with the amplitude and phase parts individually and extract one after the other.

In the first step, to extract the amplitude information, we calculate the probability $W\left(x ; t \mid b, 1_{\mathbf{k}}\right)$ of finding the atom at position $x$ in the standing wave given that we have detected a spontaneously emitted photon at time $t$ in the reservoir mode of wave vector $\mathbf{k}$ and that the atom is in its internal state $|b\rangle$. We find this probability from the reduced state 


$$
\left|\psi_{b, 1_{\mathbf{k}}}^{(\text {atom })}\right\rangle=\mathcal{N}\left\langle 1_{\mathbf{k}}\left|\langle b \mid \Psi(t)\rangle=\mathcal{N} \int \mathrm{d} x f(x) C_{b, 1_{\mathbf{k}}}(x ; t)\right| x\right\rangle
$$

of the atom, where $\mathcal{N}$ is a normalization factor. We note that the reduced state depends, via the coefficients $C_{b, 1_{\mathbf{k}}}(x, t)$, on the outcome of the measurement. Hence, the conditional position probability, for $t \gg \gamma^{-1}$, is given by

$$
W\left(x ; \infty \mid b, 1_{\mathbf{k}}\right)=\left|\left\langle x \mid \psi_{b, \mathbf{1}_{\mathbf{k}}}^{(\text {atom) }}\right\rangle\right|^{2}=\mathcal{F}\left(x, \delta_{k}\right)|f(x)|^{2},
$$

where the filter function has the form

$$
\begin{aligned}
\mathcal{F}\left(x, \delta_{k}\right) & =|\mathcal{N}|^{2}\left|C_{b, 1_{\mathbf{k}}}(x, \infty)\right|^{2} \\
& =|\mathcal{N}|^{2} \frac{\left|G_{k_{0}}\right|^{2} \delta_{k}^{2}}{\left[G^{2} \sin ^{2}(\kappa x)-\delta_{k}^{2}\right]^{2}+\delta_{k}^{2} \gamma^{2}}
\end{aligned}
$$

Note that we have replaced $G_{\mathbf{k}}$ in the expression for $\mathcal{F}\left(x, \delta_{k}\right)$ by its value $G_{k_{0}}$ at $k_{0}=\omega_{a b} / c$, which is a reasonable approximation in the region of interest. As a consequence, the conditioned position distribution depends only on the frequency $\delta_{k}$ of the spontaneously emitted photon. It is clear from Eq. (10) that for $\gamma \ll G$, the function $\mathcal{F}\left(x, \delta_{k}\right)$ is sharply peaked at those values of $x$ that satisfy

$$
\sin (\kappa x)= \pm \delta_{k} / G
$$

and is zero everywhere else. The most general solutions of this equation are given by

$$
\begin{aligned}
& x_{n}^{+}=+\frac{1}{\kappa} \sin ^{-1}\left(\delta_{k} / G\right)+\frac{\pi n}{\kappa}, \\
& x_{n}^{-}=-\frac{1}{\kappa} \sin ^{-1}\left(\delta_{k} / G\right)+\frac{\pi n}{\kappa},
\end{aligned}
$$

where $n$ ranges over the set of integers $0, \pm 1, \pm 2, \ldots$ The superscripts \pm in Eqs. (12) and (13) correspond to the \pm signs in the transcendental Eq. (11). Thus, there are two series of points where the filter function is sharply peaked. The points in a given series are spaced by $\pi / \kappa$ from each other. The distance between the two nearest points from different series (for example, $x_{0}^{-}$and $x_{0}^{+}$) is $2 \kappa^{-1} \sin ^{-1}\left(\delta_{k} / G\right)$. A point worth mentioning is that both the series represented by $x_{n}^{ \pm}$ range over both positive and negative values on the $x$ axis. Therefore, the \pm signs in the superscripts are not to be taken to mean the sign of $x$, rather it is the sign of the offset from the origin for $n=0$.

It is instructive to note that the height of the peaks for the filter function at the values of $x=x_{n}^{ \pm}$are independent of $x$. The filter function $\mathcal{F}\left(x, \delta_{k}\right)$ can therefore be approximated, apart from a trivial factor, by $\delta$ functions at these points. As a result, we can simplify Eq. (9) as

$$
\begin{aligned}
W\left(x ; \infty \mid b, 1_{\mathbf{k}}\right) \simeq & \left.\left|\mathcal{N}^{\prime}\right|\right|^{2}\left(\cdots+\left|f\left(x_{-1}^{-}\right)\right|^{2} \delta_{x, x_{-1}^{-}}+\left|f\left(x_{0}^{-}\right)\right|^{2} \delta_{x, x_{0}^{-}}\right. \\
& +\left|f\left(x_{1}^{-}\right)\right|^{2} \delta_{x, x_{1}^{-}}+\cdots+\cdots \\
& +\left|f\left(x_{-1}^{+}\right)\right|^{2} \delta_{x, x_{-1}^{+}}+\left|f\left(x_{0}^{+}\right)\right|^{2} \delta_{x, x_{0}^{+}} \\
& \left.+\left|f\left(x_{1}^{+}\right)\right|^{2} \delta_{x, x_{1}^{+}}+\cdots\right) .
\end{aligned}
$$

Thus, determination of $W\left(x ; \infty \mid b, 1_{\mathbf{k}}\right)$ enables us to find $|f(x)|$ at the points $x=x_{n}^{ \pm}$. Note that the normalization factor is modified to $\mathcal{N}^{\prime}$ to incorporate the trivial multiplicative factors arising after the introduction of the Kronecker delta symbols for the filter function.

In the next set of measurements, we shift the standing wave with respect to the incoming atomic wave by a small amount $\epsilon$ and then calculate $W\left(x ; \infty \mid b, 1_{\mathbf{k}}\right)$. This yields the values of $|f(x)|$ at $x=x_{n}^{ \pm}+\epsilon$. Repeating the process a number of times allows us to obtain $|f(x)|$ at spatial intervals of $\epsilon$. One only needs to repeat the process a small number of times to cover the range in between the mesh points $x_{n}^{ \pm}$. Thus the resolution of the method is governed by the smallest of the three entities-the spatial shift $\epsilon$, distance between the close-by points within a series $\pi / \kappa$, and the distance between the close-by points of the two series $2 \kappa^{-1} \sin ^{-1}\left(\delta_{k} / G\right)$. These parameters depend on various experimental factors and is outside the scope of this paper. The detailed account will be given elsewhere.

We have seen how the conditional probability distribution $W\left(x ; \infty \mid b, 1_{\mathbf{k}}\right)$ can be used to determine the amplitude of the center-of-mass wave function $f(x)$. However, complete determination of $f(x)$ requires information about both the amplitude and the phase. In order to determine the phase $\varphi$ [with $f(x)=|f(x)| \exp (i \varphi)$ ], we consider the conditional momentum distribution

$$
\begin{aligned}
\widetilde{W}\left(p ; t \mid b, 1_{\mathbf{k}}\right) & =\left|\left\langle p \mid \psi_{b, 1_{\mathbf{k}}}^{\text {(atom) }}\right\rangle\right|^{2} \\
& =\frac{|\mathcal{N}|^{2}}{2 \pi \hbar}\left|\int \mathrm{d} x f(x) C_{b, 1_{\mathbf{k}}}(x ; t) \mathrm{e}^{-i p x / \hbar}\right|^{2}
\end{aligned}
$$

of the atom. To note, this relation (15) is obtained from the reduced atomic state of Eq. (8) via a projection onto momentum eigenstates $|p\rangle$ through the relation $\langle p \mid x\rangle$ $=(2 \pi \hbar)^{-1 / 2} \exp (-i p x / \hbar)$. In the steady state $\left(t \gg \gamma^{-1}\right)$, Eq. (15) can be rewritten as

$$
\begin{gathered}
\widetilde{W}\left(p ; \infty \mid b, 1_{\mathbf{k}}\right)=\frac{|\mathcal{N}|^{2}}{2 \pi \hbar}\left|\int \mathrm{d} x\right| f(x)|| C_{b, 1_{\mathbf{k}}}(x ; \infty) \mid \\
\times \mathrm{e}^{-\left.i[p x / \hbar-\varphi(x)+\mu(x)+k x \cos \theta)\right|^{2},}
\end{gathered}
$$

where

$$
\mu(x)=\tan ^{-1}\left(\frac{\delta_{k} \gamma}{G^{2} \sin ^{2}(\kappa x)-\delta_{k}^{2}}\right) .
$$


Here, for the sake of simplicity, we have assumed $G_{\mathbf{k}}$ to be real. For $\gamma \ll G$, the steady-state probability amplitude $\left|C_{b, 1_{k}}(x, \infty)\right|$ is a sharply peaked function at $x_{n}^{ \pm}$, as discussed earlier. Thus, the expression for the momentum distribution $\widetilde{W}\left(p ; \infty \mid b, 1_{\mathbf{k}}\right)$ can be simplified considerably to obtain

$$
\begin{aligned}
\widetilde{W}\left(p ; \infty \mid b, 1_{\mathbf{k}}\right)= & \frac{\left|\mathcal{N}^{\prime}\right|^{2}}{2 \pi \hbar}\left|\sum_{n}\right| f\left(x_{n}^{+}\right) \mid \mathrm{e}^{-i\left[\chi\left(p, x_{n}^{+}\right)-\varphi\left(x_{n}^{+}\right)\right]} \\
& +\left.\left|f\left(x_{n}^{-}\right)\right| \mathrm{e}^{-i\left[\chi\left(p, x_{n}^{-}\right)-\varphi\left(x_{n}^{-}\right)\right]}\right|^{2}
\end{aligned}
$$

where

$$
\chi(p, x)=p x / \hbar+\mu(x)+k x \cos \theta .
$$

Here we note that $\mu(x)$ only takes values $+\pi / 2$ or $-\pi / 2$ at $x_{n}^{ \pm}$depending on the sign of $\delta_{k}$ and the gradient of $\sin (x)$. Also note the modified normalization factor which incorporates the multiplicative factors arising after the introduction of the Kronecker delta symbols. It is clear from Eq. (18) that amplitudes corresponding to the wave function at different positions, $x_{n}^{ \pm}$, interfere. We know, in principle, all the quantities in Eq. (18) except the phases $\varphi\left(x_{n}^{ \pm}\right)$. The question is how to recover the phase information from these interference terms. From the amplitude measurement exercise, we already know the position interval, say $\left[x_{\min }, x_{\max }\right]$, in which the wave function differs substantially from zero. Outside this region, wave-function amplitude is negligible and there is no need to recover the corresponding phase information. The extent of this significant region determines the limiting value of $n$ and the number of terms to be considered under the summation sign in Eq. (18). A careful look at Eq. (18) also shows that after expanding the terms under the summation sign, the resulting expression not only contains the phase differences at the nearest-neighboring points, i.e., $x_{n}^{ \pm}$and $x_{n-1}^{ \pm}$, but it also includes all possible combinations of phase differences over the whole range of $x_{n}^{ \pm}$. However, we only need the phase differences for the nearest-neighboring points. To determine the unknown phase differences from the interference terms, we need to measure corresponding set of values of the momentum distribution for different momenta $p$. These values should be chosen randomly such that the coefficient matrix of the resulting set of nonlinear equations has a nonsingular determinant. In order to recover the individual phases from the measured phase differences, we can assume some arbitrary initial phase. Thus it is possible to reconstruct the original wave function up to an arbitrary phase factor. To obtain the phases at another set of positions $x_{n}^{ \pm}+\epsilon$, we shift the standing wave by $\epsilon$ with respect to the incoming wave and repeat the process again. Thus, by making a number of measurements for the momentum distribution and solving the resulting set of algebraic equations, one can recover the phase information of the atomic wave function in the full interval $\left[x_{\min }, x_{\max }\right]$.

Note that we have discussed a method to measure the atomic wave function at discrete grid points separated by the resolution limit of the given experimental setup. Thus, resolution of the reconstructed wave function will depend upon the precision with which the various parameters can be controlled experimentally. The relevant experimental and numerical issues will be discussed elsewhere. We would also like to point out that measurement of the wave function is conditioned on the detection of the frequency and the direction of the spontaneously emitted photon. Moreover, due to the isotropic nature of the spontaneously emitted radiation one needs $4 \pi$ detectors for efficient reconstruction. However, it is not necessary to measure all the atoms from a given atomic beam. In real experimental situation, only atoms whose spontaneously emitted photons have indeed been detected can be considered while ignoring the others.

\section{CONCLUSION}

To summarize, we have shown that Autler-Townes spectroscopy comes in as a handy tool while measuring the center-of-mass wave function of an atomic beam. We have further shown that manipulating the internal degrees of freedom of an atom through the cavity field allows us to characterize its center-of-mass motion. The scheme is based on the fact that the spontaneously emitted photons carry the information about the position and momentum distribution of an atom that is interacting in a position-dependent manner with a classical standing light field. The information about the amplitude of the wave function can be obtained from the position distribution by a series of measurements. The most essential information, i.e., the phase of the wave function is encoded in the interference term in the momentum distribution and can be extracted by repeated measurements of the momentum distribution of the atom.

\section{ACKNOWLEDGMENTS}

The authors gratefully acknowledge the support from Air Force Research Laboratories (Rome, New York), TAMU Telecommunications and Informatics Task Force (TITF) initiative, DARPA-QuIST, and the Office of Naval Research. One of us (S.Q.) would like to thank the Ministry of Science and Technology, Government of Pakistan for financial support.
[1] A. Peres, Am. J. Phys. 52, 644 (1984); W. Pauli, General Principles of Quantum Mechanics (Springer, Berlin, 1980); H. Reichenbach, Philosophical Foundation of Quantum Mechanics (University of California Press, Berkeley, CA, 1946) Sec. 20; W. Gale, E. Guth, and G.T. Trammell, Phys. Rev. 165,
1434 (1968); W. Band and J.L. Park, Found. Phys. 1, 133 (1970).

[2] J. Mod. Opt. 11/12 (1997), special issue on quantum state preparation and measurement, edited by W.P. Schleich, M.G. Raymer, and U. Leonhardt, Measuring the Quantum State of 
Light (Cambridge University Press, Cambridge, London, 1997).

[3] E.P. Wigner, Phys. Rev. 40, 749 (1932); M. Hillery, R.F. O'Connell, M.O. Scully, and E.P. Wigner, Phys. Rep. 106, 121 (1984).

[4] K. Vogel and H. Risken, Phys. Rev. A 40, 2847 (1989).

[5] G.M. D'Ariano, U. Leonhardt, and H. Paul, Phys. Rev. A 52, R1801 (1995); U. Leonhardt, H. Paul, and G.M. D'Ariano, ibid. 52, 4899 (1995); M. Ahmad, S. Qamar, and M.S. Zubairy, ibid. 62, 043814 (2000); ibid. 64, 023811 (2001).

[6] M.S. Zubairy, Phys. Lett. A 222, 91 (1996); M.S. Zubairy, Phys. Rev. A 57, 2066 (1998); T. Azim and M.S. Zubairy, Phys. Lett. A 250, 344 (1998); M. Mahmoudi, H. Tajalli, and M.S. Zubairy, J. Opt. B: Quantum Semiclassical Opt. 2, 315 (2000); T. Azim, S. Qamar, and M.S. Zubiary, J. Mod. Opt. 49, 245 (2002).

[7] M. Brune, S. Haroche, V. Lefevre, J.M. Raimond, and N. Zagury, Phys. Rev. Lett. 65, 976 (1990).

[8] H. Paul, P. Torma, T. Kiss, and I. Jex, Phys. Rev. Lett. 76, 2464 (1996); M.G.A. Paris, Phys. Rev. A 53, 2658 (1996); K. Banaszek and K. Wodkiewicz, Phys. Rev. Lett. 76, 4344 (1996); C.T. Bodendorf, G. Antesberger, M.S. Kim, and H. Walther, Phys. Rev. A 57, 1371 (1998); L.G. Lutterbach and L. Davidovich, Phys. Rev. Lett. 78, 2547 (1997).

[9] D. Leibfried, D.M. Meekhof, B.E. King, C. Monroe, W.M. Itano, and D.J. Wineland, Phys. Rev. Lett. 77, 4281 (1996); D. Leibfried, D.M. Meekhof, C. Monroe, B.E. King, W.M. Itano, and D.J. Wineland, J. Mod. Opt. 44, 2485 (1997); P.J. Bardroff, M.T. Fontenelle, and S. Stenholm, Phys. Rev. A 59, R950 (1999).

[10] P.J. Bardroff, C. Leichtle, G. Schrade, and W.P. Schleich, Phys.
Rev. Lett. 77, 2198 (1996); W. Vogel, D.-G. Welsch, and L. Leine, J. Opt. Soc. Am. B 4, 1633 (1987).

[11] C. Iaconis and I.A. Walmsley, Opt. Lett. 21, 1783 (1996); D.A. Kokorowski and D.E. Pritchard, J. Mod. Opt. 44, 2575 (1997); S.H. Kienle, D. Fischer, W.P. Schleich, V.P. Yakovlev, and M. Freyberger, Appl. Phys. B: Lasers Opt. B65, 735 (1997); M. Freyberger, S.H. Kienle, and V.P. Yakovlev, Phys. Rev. A 56, 195 (1997); S.H. Kienle, D.G. Fischer, and M. Freyberger, ibid. 60, 1471 (1999).

[12] T.J. Dunn, J.N. Sweetser, I.A. Walmsley, and C. Radzewicz, Phys. Rev. Lett. 70, 3388 (1993); T.J. Dunn, I.A. Walmsley, and S. Mukamel, ibid. 74, 884 (1995).

[13] C. Leichtle, W.P. Schleich, I.Sh. Averbukh, and M. Shapiro, Phys. Rev. Lett. 80, 1418 (1998); T.C. Weinacht, J. Ahn, and P.H. Bucksbaum, ibid. 80, 5508 (1998).

[14] X. Chen and J.A. Yeazell, Phys. Rev. A 56, 2316 (1997).

[15] M.S. Chapman, T.D. Hammond, A. Lenef, J. Schmiedmayer, R.A. Rubenstein, E. Smith, and D.E. Pritchard, Phys. Rev. Lett. 75, 3783 (1995); S. Durr, T. Nonn, and G. Rempe, Nature (London) 395, 33 (1998); M. Kasevich and S. Chu, Phys. Rev. Lett. 67, 181 (1991); F. Riehle, Th. Kisters, A. Witte, J. Helmcke, and Ch.J. Borde, ibid. 67, 177 (1991).

[16] A.P. Kasantsev, G.I. Surdutovich, and V.P. Yakovlev in Mechanical Action of Light on Atoms (World Scientific, Singapore, 1990).

[17] S.H. Autler and C.H. Townes, Phys. Rev. 100, 703 (1955); P.L. Knight and P.W. Milonni, Phys. Rep. 66, 21 (1980).

[18] A.M. Herkommer, W.P. Schleich, and M.S. Zubairy, J. Mod. Opt. 44, 2507 (1997).

[19] S. Qamar, S.-Y. Zhu, and M.S. Zubairy, Opt. Commun. 176, 409 (2000). 